\title{
Clinical significance of positive Raoultella ornithinolytica and Staphylococcus hominis cultures in a post lobectomy patient. A case report
}

\author{
Evaggelina Papakanderaki, Konstantinos Kanakakis, Sotirios Goule, Maria Chounti, \\ Panagiotis Hountis
}

Department of Cardiothoracic Surgery, Athens Naval and Veterans Hospital, Athens, Greece

\begin{abstract}
Raoultella ornithinolytica is an encapsulated, Gram-negative, nonmotile aerobic rob which was reclassified from Klebsiella genus belonging to the family of Enterobacteriaceae. It is a rare human infection and few cases have been reported in post thoracotomy patients. Here we present a case of a left lower lobectomy patient that was complicated by pleural effusion, high fever with positive sputum cultures of Raoultella ornithinolytica and positive pleural fluid cultures of Staphylococcus hominis. The disease is related with aquatic life poisoning. There are few cases reported and even fewer postoperatively. The infection is rare in humans therefore the bacteria is still underreported.
\end{abstract}

\section{Introduction}

Raoultella ornithinolytica (R0) is an encapsulated, Gram-negative, nonmotile aerobic rob which belongs to the Enterobacteriaceae family. Its name is a result of the ability of $R$. ornithinolytica to produce ornithine decarboxylase. It was assorted from Klebsiella and it is related with fish poisoning and aquatic environments. These bacteria are found in the natural environment: plants, water, soil and insects. RO is a rare human infection and only small number of patients have been reported postoperatively. Fewer cases are reported that relate to pulmonary challenged patients [1]. It is observed that

Corresponding Author: Panagiotis G. Hountis, Department of Cardiothoracic Surgery, Athens Naval and Veterans Hospital Deinokratous 70, 11521 Athens, Greece. Tel. +30.6986004089 - Fax +30.210 .2133437 .

E-mail:panos_hountis@hotmail.com

Key words: Raoultella ornithinolytica; Staphylococcus hominis; lung cancer; lobectomy; thoracic surgery.

Received for publication: 19 August 2017

Accepted for publication: 28 January 2018

CCopyright E. Papakanderaki et al., 2018

Tipografia PI-ME Editrice, Italy

Monaldi Archives for Chest Disease 2018; 88:885

doi: 10.4081/monaldi.2018.885

This article is distributed under the terms of the Creative Commons Attribution Noncommercial License (by-nc 4.0) which permits any noncommercial use, distribution, and reproduction in any medium, provided the original author(s) and source are credited. the effects of RO are greater and more frequent in immunosuppressed and especially in oncological patients. On the other hand coagulase negative staphylococci (CoNS) - as Staphylococcus hominis (SH) are a diverse group of Gram-positive cocci, commonly isolated from human skin as normal flora, and recently recognised as a significant cause of nosocomial bloodstream infections. Indwelling medical devices and critically ill patients have been recognised as the main parameters that rise the number of reported cases. CoNS account for $12 \%-80 \%$ mortality in hospitalised patients and also $16 \%$ morbidity has been associated with CoNS bacteraemia [2.] Here we present a case of postoperative fever and positive sputum culture of RO as well as $\mathrm{SH}$ cultures of pleural fluid without proven bacteremia, after left lower lobectomy due to non-small cell lung cancer (NSCLC).

\section{Case Report}

A 75-year-old male patient with a lung tumor in the inferior left lobe was admitted to our hospital with hemoptysis. He had a history of heavy smoking for about 50 years ( 100 pack/years). The patient had a history of femoral-popliteal by pass in the right leg and a dualchamber, rate-modulated pacemaker (DDDR) three and two years ago respectively. During the diagnostic workup an irregular pulmonary left lower shadow possibly representing a mass was identified. Fine needle biopsy (FNB) under CT guidance that was performed was positive for NSCLC. A PET-CT scan showed that there were no other suscpicious foci but a dental area was illuminated and this was clinically correlated with an infectious process (Figure 1). Antibiotics were started blindly and after a 7-day course on Amoxicilline clavulanate $625 \mathrm{mgx} 3$ we performed a left lower lobectomy with a double endotracheal tube. He had an uneventful postoperative period for 3 days and in the $4^{\text {th }}$ post-operative day all chest tubes were removed. In the $6^{\text {th }}$ postoperative day the patient had progressively advanced high temperature up to $38^{\circ} \mathrm{C}$ accompanied with cough, malaise and dyspnea. His ability to mobilize himself was deteriorated. His laboratory exams showed WBC $11.43 \mathrm{~K} / \mu \mathrm{l}$ (83\% neutrophils) and a CRP 271 (0-7 $\mathrm{mg} / \mathrm{l})$. Due to enteric paresis, a gas tube and enema were inserted. Samples were taken from sputum and pleural fluid for microbiological analysis. The sputum sample was positive for Raoultella ornithinolytica, whereas the pleural fluid sample was positive for Staphylococcus hominis. The treatment was changed to Meropenem 1 grx3 and Ciprofloxacin $400 \mathrm{mgx} 3$ in relation with the antibiogram. Moreover, a drainage tube was inserted to extract the pleural effusion (Figure 2). The patient was progressively improving and was discharged in the $20^{\text {th }}$ postoperative day, having a good clinical condition. The treatment continued with Ciprofloxacin 500 mgx2. Blood cultures were negative. 


\section{Discussion}

In 1989, Sakazaki et al. described the histamine-producing ornithine-positive Klebsiella oxytoca and renamed it 'Klebsiella ornithinolytica' [3]. This unique bacterium was later named as R0; Kanki et al. concluded that $R$. ornithinolytica strains and $R$. planticola are isolated at high frequencies from raw fish and have always been the most important histamine-producing bacteria causing histamine poisoning, so-called 'Scombroid Fish Poisoning'. R0 has also been isolated from the gut of fish such as tuna, bonito, sardine, and mackerel, ticks, termites and from estuarine water. Its ability to colonize the inner surfaces of indwelling catheters is a major cause of hospital-acquired infections. These mechanisms as well as its ability to form biofilms could play an important role in the environmental dissemination of this bacterium.

With respect to antibiotic susceptibility, RO has been known to be resistant to ampicillin, similar to our findings, and produces chromosomally-encoded class A beta-lactamases [4].

RO has been reported in various different clinical situations mainly in immunosuppresed patients. Some immunocompetent cases of Roultella ornithinolytica bacteremia [5] have also been reported. Its isolation must always be considered as an important complication although the prognosis of the patients is quite satisfactory.

In a study by Haruki et al. [5] the most common infectious focus was biliary infection in elderly patients with a history of biliary inter-

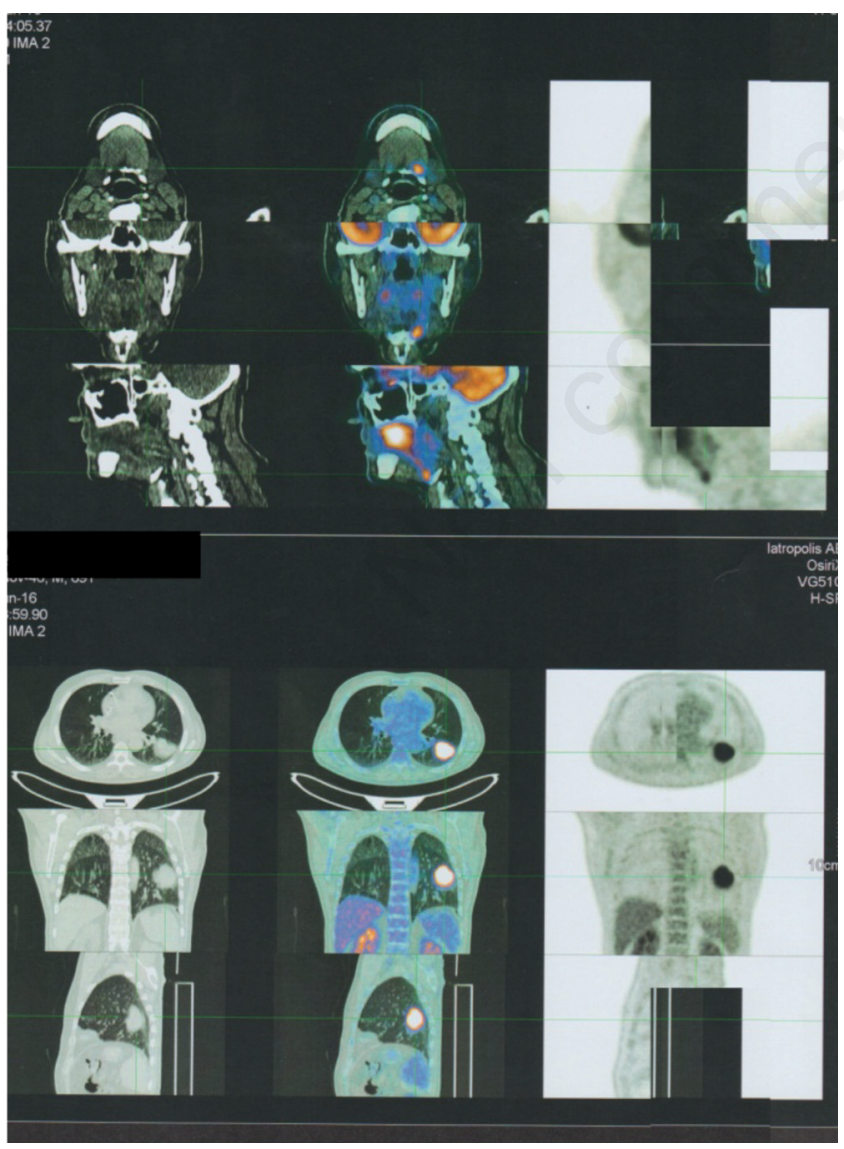

Figure 1. Positron Emission Tomography-CT scan of the patient with the inflammatory loci in the dental area as well as the neoplasmatic loci in the lung. vention or malignancy. These patients were considered to be at a great risk for this particular infection. Septic arthritis and sepsis in general $[6,7]$ is possibly a major clinical consequence of this bacterial infection $[8,9]$. Children, especially in immunocompromised conditions are vulnerable in manifesting clinically significant complicated infections [10].

In a retrospective study by Boattini M. et al. Raoultella spp. were deemed responsible for clinical infections with RO presented as cystitis in $36 \%$ of the cases and pneumonia in $24 \%$ [11]. The findings of Chun's et al. study [12] were that $R$. ornithinolytica bacteremia occurs mainly in patients with underlying malignancies [13]. The overall outcome was not favorable, despite favorable antimicrobial susceptibility test results. The findings of this study contradicted those of other studies that demonstrated that infections from Raoultella species have good prognoses.

In a retrospective study from four university hospital centres and $187 R$. ornithinolytica isolates this was associated with diseases such as osteomyelitis, meningitis, cerebral abscess, mediastinitis, pericarditis, conjunctivitis, and otitis [14,15]. Also, a case report has been reported after pancreaticoduodenectomy [16] as well as in diabetic limbs [17]. It has been also isolated by human saliva and in oral microbiota of healthy patients as well as dentin of infected root canals [18]. Regarding the several conditions studied, there was a higher prevalence in patients with gastritis and patients with periodontal disease [19].

SH on the other hand has been traditionally considered as a saprophytic bacterium of low virulence and a part of the normal human skin flora. Although studies have shown that clinical criteria apply as well and in cases that patient condition deteriorates it should be treated promptly after antibiotic susceptibility testing. Oncological patients, immunosuppresed and patients in ICU as well as septic conditions may predispose to the high possibility that this innocent bacterium may cause serious complications [20]. Our patient was diagnosed immediately after tube removal with pleural effusion and high fever without any sign of rashes or other histamine reaction. We suppose that this infection might be attributed to a residual contaminated parapneumonic fluid. This hypothesis is further proved by the finding of a collection in the pleural cavity, when symptoms first appeared, without any lung involvement. $R$. ornithinolytica should never be considered simply a saprophytic bacterium that occasionally contaminates bronchial lavage or other deep respiratory samples or surgical sites. Regarding our patient if this infection was ignored it might have led to devasting consequences. To the best of our knowledge such a combination in a post lobectomy patient is the first to be reported in the literature.

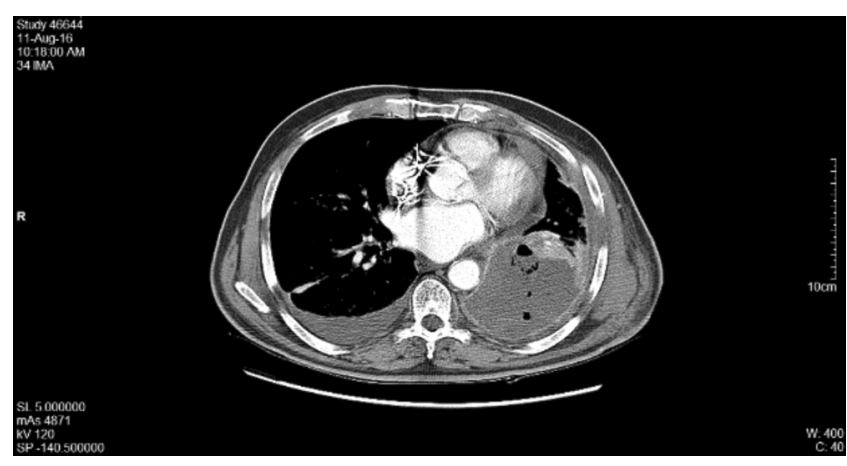

Figure 2. Computerized tomography of the lung showing the postoperative pleural effusion of the left hemithorax. 


\section{References}

1. Sękowska A, Dylewska K, Gospodarek E, Bogiel T. Catheter-related blood stream infection caused by Raoultella ornithinolytica. Folia Microbiol (Praha) 2015;60:493-5.

2. Gautam V, Sethuraman N, Kaur R, et al. Changing epidemiology of coagulase-negative staphylococci in normal flora of skin. Indian $\mathrm{J}$ Med Microbiol 2017;35:277-8.

3. Sakazaki R, Tamura K, Kosako Y, Yoshizaki E. Klebsiella ornithinolytica sp. nov., formerly known as ornithine-positive Klebsiella oxytoca. Curr Microbiol 1989;18:201-6.

4. Kanki M, Yoda T, Tsukamoto T, Shibata T. Klebsiella pneumonia produces no histamine: Raoultella planticola and Raoultella ornithinolytica strains are histamine producers. Appl Environ Microbiol 2002;68:3462-6.

5. Haruki Y, Hagiya H, Sakuma A, et al. Clinical characteristics of Raoultella ornithinolytica bacteremia: a case series and literature review. J Infect Chemother 2014;20:589-91.

6. Sueifan M, Moog V, Rau E, Eichenauer T. Sepsis caused by Raoultella ornithinolytica in an immunocompetent patient. Anaesthesist 2016;65:129-33.

7. Kaya S, Bayramoğlu G, Sönmez M, Köksal İ. Raoultella ornithinolytica causing fatal sepsis. Braz J Infect Dis 2015;19:230-1.

8. Seng P, Theron F, Honnorat E, et al. Raoultella ornithinolytica: An unusual pathogen for prosthetic joint infection. ID Cases 2016;25;5:46-8.

9. Venus K, Vaithilingam S, Bogoch II. Septic arthritis of the knee due to Raoultella ornithinolytica. Infection 2016;44:691-2
10. Yamakawa K, Yamagishi Y, Miyata K, et al. Bacteremia caused by Raoultella ornithinolytica in two children. Pediatr Infect Dis J 2016;35:452-3.

11. Boattini M, Almeida A, Cardoso C, et al. Infections on the rise: Raoultellaspp., clinical and microbiological findings from a retrospective study. Infect Dis (Lond) 2016;48:87-91.

12. Chun S, Yun JW, Huh HJ, Lee NY. Clinical characteristics of Raoultella ornithinolytica bacteremia. Infection 2015;43:59-64.

13. Hadano Y, Tsukahara M, Ito K, et al. Raoultella ornithinolytica bacteremia in cancer patients: report of three cases. Intern Med 2012;51:3193-5.

14. Seng P, Boushab BM, Romain F, et al. Emerging role of Raoultella ornithinolytica in human infections: a series of cases and review of the literature. Int J Infect Dis 2016;45:65-71.

15. Schmutz N, Adler T, Schelhorn N, et al. Postoperative osteomyelitis of a distal phalanx caused by Raoultella Ornithinolytica. Handchir Mikrochir Plast Chir 2016;48:175-8.

16. Bhatt P, Tandel K, Das NK, Rathi KR. New Delhi metallo- $\beta$-lactamase producing extensively drug resistant Raoultella ornithinolytica isolated from drain fluid following Whipple's pancreaticoduodenectomy. Med J Armed Forces India 2015;71(S2):S609-11.

17. Kabbara WK, Zgheib YR. Diabetic foot infection caused by Raoultella ornithinolytica. Am J Health Syst Pharm 2015;15;72:2147-9.

18. Nakajo K, Nakazawa F, Iwaku M, Hoshino E. Alkali-resistant bacteria in root canal systems. Oral Microbiol Immunol 2004;19:3904.

19. Heggendorn FL, Gonçalves LS, Dias EP, et al. Detection of sulphate-reducing bacteria in human saliva. Acta Odontol Scand 2013;71:1458-63.

20. Pradipta IS, Sodik DC, Lestari K, et al. Antibiotic resistance in sepsis patients: evaluation and recommendation of antibiotic use. N Am J Med Sci 2013;5:344-52. 